\title{
Blast-Induced Tinnitus and Hyperactivity in the Auditory Cortex of Rats
}

Hao Luo ${ }^{1}$, Edward Pace ${ }^{1}$ and Jinsheng Zhang ${ }^{1,2}$

1. Department of Otolaryngology-Head and Neck Surgery, Wayne State University School of Medicine, Detroit, Michigan

2. Department of Communication Sciences \& Disorders, Wayne State University College of Liberal Arts \& Sciences, Detroit, Michigan

Running head: Blast-induced tinnitus and neural activity changes

Number of figures: 4

*Correspondence: Dr. Jinsheng Zhang, Otolaryngology-Head and Neck Surgery, 5E-UHC, Wayne State University School of Medicine, 4201 Saint Antoine, Detroit, Michigan 48201, U.S.A.; Phone: (313) 577 0066; Fax: (313) 577 6318; E-mail: jinzhang@ med.wayne.edu.
Abbreviations:
$\mathrm{ABR}$
$\mathrm{AC}$
auditory brainstem response
$\mathrm{CF}$
auditory cortex
DCN
characteristic frequency
FTC
dorsal cochlear nucleus
IC
frequency tuning curve
SFRs
inferior colliculus
spontaneous firing rates 


\section{Abstract}

Blast exposure can cause tinnitus and hearing impairment by damaging the auditory

3 periphery and direct impact to the brain, which trigger neural plasticity in both auditory and non-

4 auditory centers. However, the underlying neurophysiological mechanisms of blast-induced

5 tinnitus are still unknown. In this study, we induced tinnitus in rats using blast exposure and

6 investigated changes in spontaneous firing and bursting activity in the auditory cortex at one day,

7 one month, and three months after blast exposure. Our results showed that spontaneous activity

8 in the tinnitus positive group began changing at one month after blast exposure, and manifested

9 as robust hyperactivity at all frequency regions at three months after exposure. We also observed

10 an increased bursting rate in the low frequency region at one month after blast exposure and in

11 all frequency regions at three months after exposure. Taken together, spontaneous firing and

12 bursting activity in the auditory cortex played an important role in blast-induced chronic tinnitus

13 as opposed to acute tinnitus, thus favoring a bottom-up mechanism.

14

15 Keywords: Blast, Tinnitus, Traumatic brain injury (TBI), Auditory cortex, Spontaneous activity, 16 Bursting, Rat 


\section{Introduction}

Tinnitus, the perception of sound without an external source, is a complex condition occurring in an estimated $10-15 \%$ of the American population (Henry et al., 2005). While there are many causes of tinnitus, high-pressure blast shock waves are often the ones that contribute to both tinnitus and hearing loss in military service members (Geckle and Lee, 2004). In addition to the audiological impact, blast exposure can lead to traumatic brain injury, with one study observing that over $53 \%$ of patients recovering from traumatic brain injury developed tinnitus symptoms (Jury and Flynn, 2001). Previous studies have indicated that the occurrence of both injury and compensatory plastic changes in central auditory structures after blast exposure may trigger tinnitus development (Cave et al., 2007, Mao et al., 2012). Nevertheless, there is still a lack of effective treatments for blast-induced tinnitus, largely because of a limited understanding of its underlying neuropathophysiology.

Over the last two decades, numerous studies have suggested that noise-induced tinnitus is correlated with changes in spontaneous activity, neurosynchrony and maladaptive plasticity in the auditory system following acoustic trauma (Bauer, 2004, Eggermont and Roberts, 2004, Eggermont, 2007, Roberts et al., 2010, Eggermont and Tass, 2015, Wu et al., 2016). Increased spontaneous firing rates (SFRs) of neurons in the central auditory system are one potential neural substrate of tinnitus (Eggermont and Roberts, 2004, Wu et al., 2016). Specifically, an increase in SFRs has been found in the dorsal cochlear nucleus (DCN) (Zhang and Kaltenbach, 1998, Wu et al., 2016), ventral cochlear nucleus (Vogler et al., 2011), inferior colliculus (IC) (Bauer et al., 2008) and auditory cortex (AC) (Norena and Eggermont, 2003, Llano et al., 2012). In addition, spontaneous bursting in the auditory system has been suggested to subserve tinnitus (Roberts et al., 2010, Wu et al., 2016). For example, it has been reported that chronic increases in bursting 

activity occurs in the auditory nerve (Liberman and Kiang, 1978), DCN (Finlayson and Kaltenbach, 2009, Wu et al., 2016) and IC (Chen and Jastreboff, 1995) following noise trauma.

3 However, it is not clear whether noise trauma induces a lasting increase of bursting activity in the AC (Norena and Eggermont, 2003). Furthermore, it remains to be determined whether blastinduced tinnitus shares the same neural mechanisms as noise-induced tinnitus.

Although tinnitus appears to result from aberrant neural activity in the auditory system (Jastreboff, 1990, Eggermont and Roberts, 2004), it is unclear which levels of the auditory system are most closely involved in tinnitus generation. Recently, our lab showed that increased spontaneous activity in the DCN and IC of rats with behavioral evidence of tinnitus occurred immediately following blast exposure and lasted for up to one month in the DCN and three

11 months in the IC (Luo et al., 2014ab). Since noise-induced tinnitus has been proposed to be 12 related to increased SFRs in the AC at both acute (Kimura and Eggermont, 1999, Norena et al., 13 2003) and chronic stages (Eggermont, 2000, Seki and Eggermont, 2003), one wonders how 14 neural activity changes in the AC manifest during blast-induced acute and chronic tinnitus. 15 Compared to activity change patterns in the DCN and IC, the information on AC activity will 16 also help delineate whether blast-induced spontaneous firing and bursting activity takes a 17 bottom-up or top-down approach during tinnitus development. In the current study, we investigated changes in SFRs and bursting activity in the AC of rats with blast-induced tinnitus and characterized the time course of the induced neural activity 20 changes. Specifically, we sought to establish how hyperactivity emerged over a three-month 21 period following blast exposure and to determine whether the magnitude of the induced 22 hyperactivity remained constant or shifted over time. Our results demonstrated robust 23 hyperactivity in all frequency regions of the $\mathrm{AC}$ in tinnitus positive rats at three months post- 

be mediated more at the brainstem level, whereas blast-induced chronic tinnitus may reside more

3 within the AC.

\section{$5 \quad$ Experimental Procedures}

\section{Animal subjects}

A total of thirty-nine adult male Sprague Dawley rats (70-80 days old at the beginning of experiments) were used for this study. It should be noted here that data collected from the DCN

9 and IC of the same animals have been published in two separate papers, and detailed experimental procedures have been provided (Luo et al., 2014ab). Briefly, the rats were divided

11 into three post-blast survival groups: one day, one month and three months post-blast exposure.

12 Each group contained nine blasted rats and four age-matched controls. Experimental rats were 13 exposed to a single 22-psi (equivalent to $197.5 \mathrm{~dB}$ peak SPL) blast with the left ear open and the 14 right ear occluded with an earplug plus mineral oil. All experimental procedures were carried out 15 by following the guidelines of the institutional Animal Care and Use Committee at Wayne State 16 University.

\section{Electrophysiology recording}

Electrophysiological recordings in the $\mathrm{AC}$ were performed in each rat to examine blast-

20 induced neural activity changes. Briefly, each animal was anesthetized with a mixture of air (1

$21 \mathrm{l} / \mathrm{min})$ and isoflurane $(5 \% \mathrm{v} / \mathrm{v})$. Before inserting the electrode, a craniotomy was performed to

22 provide access to the right $\mathrm{AC}$. To reach the AC, anatomical landmarks of the right side skull 23 were used and the dura mater above the $\mathrm{AC}$ was removed. Using a micromanipulator (Kopf 
Luo et al. Blast-induced tinnitus and neural activity changes

1 Model 1460-61), a custom-made 16-channel microwire electrode array was inserted into the AC.

2 Each electrode array consisted of 16 polyimide insulated platinum/iridium microwires. They

3 were arranged in two rows with eight wires in each row. The parameters of the array were:

4 diameter $=50 \mu \mathrm{m}$, electrode spacing $=400 \mu \mathrm{m}$, row spacing $=400 \mu \mathrm{m}$, and impedance $=20-50$

$5 \mathrm{k} \Omega$. The electrode array was inserted into the $\mathrm{AC}$ at $0.8-0.9 \mathrm{~mm}$ from its surface, which

6 corresponds to layers 4-5 of the cortex (Hughes et al., 2010). Prior to insertion, the array was

7 dipped in 3\% DiI solution (1,1'-dioctadecyl-3,3,3',3'-tetramethylindocarbocyanine perchlorate,

8 Invitrogen) prepared with N,N-Dimethylformamide (DMF) to label the electrode insertion tracks.

9 After placement of the array, the exposed cortex was covered with $2 \%$ agar to prevent

10 desiccation. The output from the headstage channels was connected to a real-time auditory

11 electrophysiological station (RZ2, TDT). The station's settings were: $25 \mathrm{kHz}$ sampling rate, 100-

$123000 \mathrm{~Hz}$ bandpass filter, and threshold at 1.5 times the standard deviation. The frequency tuning

13 curve (FTC) properties of all recording sites were determined in response to tone pips with

14 different frequency-intensity combinations. Spontaneous single- and multi-unit spikes were

15 recorded twice; once 5 min prior to and another 5 min after FTC construction. Each spontaneous

16 recording period lasted 5 minutes. Spontaneous activity data collected after FTC construction

17 were used for data analyses, since they were more stable than those collected before FTC

18 construction. At the end of each recording, the rat was euthanized and its brain removed and

19 processed histologically. The electrode tracks were later examined with the aid of a Nikon

20 fluorescent microscope (Eclipse E400).

21

22 Data analysis 

frequency of a stimulus tone at the lowest intensity level needed to evoke neuronal activity. CFs were determined using automated algorithms and, as expected, each channel produced a clearly defined CF. To avoid misrepresentation of CF-tagged spike activity caused by variable electrode

5 penetrations in the AC across different rats, data from recording sites of three adjacent frequency

6 bands based on their CFs were pooled into three separate groups. Specifically, sites with CFs at

$7 \quad 2-4 \mathrm{kHz}$ were grouped to represent a low frequency band, those with CFs at $4-16 \mathrm{kHz}$ were 8 grouped to represent a middle frequency band, and those at 16-42 kHz were grouped to represent 9 a high frequency band.

Bursting activity was detected using the Poisson-surprise method (Legendy and Salcman, 1985) with Neuroexplorer software (Nex Technological). In this method, it is assumed that a

12 spike train has a Poisson distribution, and the negative binary logarithm of the probability of the 13 occurrence of a given number of spikes in a designated time interval is considered. When the 14 Poisson-surprise value increases, spikes in a burst are added from at least three spikes. Only 15 bursts with a surprise value $\geq 4$, indicating that they occurred at least $16\left(2^{4}\right)$ times as frequently as in a Poisson spike train with the same firing rate, were considered in the analysis. as two factors, and the three frequency regions were individually tested. The distribution of the data values from each frequency band passed normality (Kolmogorov-Smirnov). Thus ANOVA 20 and multivariate analysis was used to determine significant changes. Post-hoc analyses were 21 performed using Bonferroni tests. Differences were considered to reach statistical significance 22 when $p<0.05$. 


\section{Results}

As shown in our recent study (see Figures 1-2 in (Luo et al., 2014b), there were nine tinnitus positive rats at one day following blast exposure, six tinnitus positive and three tinnitus negative rats at one month after blast, and four tinnitus positive and five tinnitus negative rats at three months after blast. Significant hearing threshold elevations were found in the exposed ears of rats at one day after blast, while rats exhibited threshold recovery at one and three months after blast exposure. In spite of threshold recovery, however, P1-N1 amplitudes were significantly reduced in both tinnitus positive and negative rats compared to control rats. This indicated that significant hearing impairment remained at certain frequencies.

\section{Changes in spontaneous firing rate}

At one day after blast exposure, there were no significant SFR differences in any frequency regions between tinnitus positive rats and control rats (Figure 1). One month after exposure, moderately decreased SFRs were found in the $16-42 \mathrm{kHz}$ region in both tinnitus positive and tinnitus negative rats compared to the controls $\left(F_{(2,49)}=4.347, p<0.05\right)$, but no significant difference was reached between tinnitus positive and control animals in betweengroup tests (one-way ANOVA with Bonferroni post-hoc tests). At three months after blast exposure, SFR changes were quite different from those found at one day and one month after exposure. The SFR in tinnitus positive rats increased in all frequency regions compared to tinnitus negative or control rats. These increases reached statistical significance at $2-4 \mathrm{kHz}\left(F_{(2,51)}\right.$ $=7.994, p<0.01), 4-16 \mathrm{kHz}\left(F_{(2,150)}=19.322, p<0.01\right)$, and 16-42 kHz $\left(F_{(2,51)}=9.384, p<\right.$ 0.01). However, no significant difference was found between tinnitus negative and control rats (one-way ANOVA with Bonferroni post-hoc tests). We also compared the SFRs of each 
1 frequency region to assess which brain region in the $\mathrm{AC}$ was more sensitive to blast exposure.

2 Our results showed significant changes at $2-4 \mathrm{kHz}\left(F_{(3,143)}=4.626, p<0.01\right), 4-16 \mathrm{kHz}\left(F_{(3,143)}\right.$

$3=4.506, p<0.01)$, and $16-42 \mathrm{kHz}\left(F_{(3,143)}=7.590, p<0.01\right)$, but there were no significant

4 differences among the status of tinnitus and recovery time points (two-way ANOVA with

5 Bonferroni post-hoc tests) (Figure 2).

6

\section{Changes in bursting rate}

As seen in Figure 3, there were no bursting rate changes in any frequency regions between tinnitus positive and control rats at one day after blast exposure. At one month after blast, bursting rates increased at the $2-4 \mathrm{kHz}$ and $4-16 \mathrm{kHz}$ regions in tinnitus positive rats. The increase was statistically significant only in the $2-4 \mathrm{kHz}$ region $\left(F_{(2,45)}=3.285, p<0.05\right)$, but there was no significant difference between tinnitus positive and control rats in between-group tests (one-way ANOVA with Bonferroni post-hoc tests). Three months after blast exposure, bursting rates in tinnitus positive rats were significantly increased across all frequency regions: $2-4 \mathrm{kHz}\left(F_{(2,51)}=4.160, p<0.05\right) ; 4-16 \mathrm{kHz}\left(F_{(2,155)}=15.471, p<0.01\right) ; 16-42 \mathrm{kHz}\left(F_{(2,51)}=\right.$ 6.173, $p<0.01$ ) (one-way ANOVA with Bonferroni tests). The significant differences were found for the within-group tests, however, and did not include the tinnitus positive and tinnitus negative rats at the $2-4 \mathrm{kHz}$ region or the tinnitus negative and control rats in any frequency regions. Similar to the analysis for SFR data, rats' tinnitus status and time points after blast exposure were used as two factors. No statistically significant differences in bursting activity change were found between frequency regions ( $>>0.05)$ (Figure 4). 


\section{Discussion}

2 The results described here extended upon our previous findings that blast-induced

3 tinnitus, observed over a three month period, is accompanied by immediate hyperactivity that

4 later shifted to lower SFRs in the DCN and by persistent hyperactivity in the IC (Luo et al.,

5 2014ab). In the current study, we found hyperactivity in the AC of tinnitus positive rats that did

6 not appear immediately after blast exposure, but later became prominent in all frequency regions

7 at three months after blast exposure. This delayed appearance of blast-induced hyperactivity in

8 the AC suggests that it became intrinsic, independent of afferent inputs from the cochlea, and 9 thus a possible basis for chronic tinnitus.

Blast-induced hyperactivity in the $A C$

The current animal study for the first time elucidated neurophysiological mechanisms

13 underlying blast-induced tinnitus in the AC. Different animal models have demonstrated that 14 noise trauma can cause an increase in SFRs and bursting activity in the AC (Norena and 15 Eggermont, 2003, 2005, 2006, Roberts et al., 2010). Such hyperactivity can occur immediately 16 after noise trauma (Norena and Eggermont, 2003) and last for several weeks (Norena and 17 Eggermont, 2006). Interestingly, we did not find increased SFR at one day and one month after 18 blast exposure. Instead, we found that SFRs increased at three months after blast exposure, but 19 only in tinnitus positive animals. Our previous studies showed that tinnitus developed one day 20 after blast exposure and was accompanied by significant elevation of SFRs in the DCN and IC.

21 These results suggest that acute tinnitus may result from hyperactivity in the lower auditory

22 brainstem, whereas chronic tinnitus may be related to higher-level auditory structures. 
Mechanistically, tinnitus may result from spontaneous activity changes in the auditory

2 system (Eggermont and Roberts, 2004), which often co-occur with a shifted balance between

3 excitatory and inhibitory inputs to tinnitus-generating neurons or neuronal populations. The

4 decrease in inhibition may result from weakened GABAergic neurotransmission, such as that

5 observed in the IC following acoustic trauma (Richardson et al., 2012). The GABAergic

6 neurotransmission could have mediated the increased SFRs in the AC. Our recent finding that

7 SFRs in the IC gradually increased after blast exposure suggests a progressive decrease in

8 inhibition (Luo et al., 2014a). Our findings are in line with the notion that tinnitus-related

9 hyperactivity at the cortical level may result from pathologically propagated gain change that is

10 originally developed in the brainstem. Abnormal spontaneous activity in the AC appears to result

11 from over-amplified spontaneous firing along the auditory pathway, which starts from the

12 cochlear nucleus (Schaette and Kempter, 2012).

Blast-induced bursting changes in the AC

Bursting firing is known to enhance the reliability of synaptic transmission and neuronal selectivity, thus triggering synaptic plasticity (Swadlow and Gusev, 2001). Bursting activity also

17 enriches functional values of cortical activity, especially regarding serial synchrony of 18 spontaneous firings in the cortex (Roberts et al., 2010). Previously, it has been reported that 19 bursting activity in the AC varies over time following noise trauma. Specifically, noise trauma 20 may induce immediate increase of burst firing, which later returns to a pre-exposure level 21 (Norena and Eggermont, 2003). However, the current study demonstrated significantly increased 22 bursting rate in the $\mathrm{AC}$ of tinnitus positive rats only at one and three months after blast exposure.

23 This increase encompassed all frequency regions at three months after blast exposure. The 

mechanisms underlying the changes in bursting activity following trauma remain unclear. It has previously been suggested that bursting activity may reflect the balance between excitation and inhibition (Krishnan and Plack, 2009). Blast exposure, through its severe mechanical impact on the cochlea and brain, may have changed bursting activity through a modification of this balance. To clearly answer this question, further investigations are needed to delineate the underlying

6 differences between noise- and blast-induced changes in bursting activity at a single cell level. Neural mechanisms of blast-induced acute and chronic tinnitus

Acoustic trauma-induced anatomical, neurochemical and neurophysiological changes in both the peripheral and central auditory system have been well studied and documented (Browne et al., 2012, Mulders and Robertson, 2013, Shore et al., 2016). Following acoustic trauma, the

12 induced cochlear damage often manifests as immediate loss of afferent inputs to portions of the 13 cochlear nuclei and higher-level structures of the auditory system. Such a rapid loss of afferent 14 inputs often results in diminished inhibition (Bilak et al., 1997, Mulders et al., 2011), which in 15 turn may cause rapid unmasking effects on complex neural circuits in the impacted structures 16 (Chambers et al., 2016). At the same time, homeostasis-related neuroplasticity occurs, which can 17 be in the forms of sprouting of new axons, development of new synapses, and modifications of 18 the neurochemical environment. These above changes may result in immediate and chronic 19 neurophysiological changes (Kaltenbach, 2011). Recently, we demonstrated immediate 20 hyperactivity one day after blast and sustained hyperactivity in the DCN and IC through one 21 month after blast exposure, although this shifted to lower SFRs in the DCN and persistent 22 hyperactivity in the IC (Luo et al., 2014ab). Together with these findings in the DCN and IC, the 23 present observations lend support to the hypothesis that, immediately after blast exposure, the 
increased spontaneous activity in the DCN and the IC may be due to hyperexcitability of neurons.

2 However, over a period of three months following blast exposure, this early stage of

3 neuroplasticity transitions to one in which elevated firing may become intrinsic to the neurons in

4 the IC or AC. Such results may support a hypothesis that acute tinnitus may result more from

5 aberrant activity in the lower-level auditory structures, while chronic tinnitus may result more

6 from abnormal neural activity in the higher-level auditory structures. In spite of this, the results

7 from the current study do not rule out the possibility that the AC is still involved in acute tinnitus.

8 Independent or combined measures of neurosynchrony and/or neurocoherence within and

9 between auditory or even non-auditory centers are needed to address the important role that

10 neural interaction and connectivity play in blast-induced neural propagation along the auditory

11 pathways (Zhang et al., 2015).

\section{Conclusions}

Our results demonstrate that blast-induced tinnitus may not involve a uniform manifestation of hyperactivity in the auditory cortex. Instead, moderately decreased spontaneous

16 firing occurred in the $\mathrm{AC}$ at one month after blast, whereas robust hyperactivity occurred across

17 all the frequency regions at three months after blast. These changes were accompanied by 18 increased bursting activity. The current findings together with those from our recent studies in 19 the DCN and IC suggest that blast-induced acute tinnitus may be related to increased neural 20 activity in the lower-level auditory centers while chronic tinnitus may be attributed to abnormal 21 neural activity in the higher-level auditory centers. Further understanding of the neural

22 mechanisms underlying blast-induced tinnitus will help develop targeted preventative and 23 therapeutic strategies. 


\section{Acknowledgements}

3

This work was supported by the Department of Defense (grant award \#W81XWH-11-20031). The authors thank Drs. Pamela VandeVord and Srini Kallakuri for their assistance during blast exposure using a shock tube in the Department of Biomedical Engineering, Wayne State University.

\section{References}

Bauer CA (2004) Mechanisms of tinnitus generation. Curr Opin Otolaryngol Head Neck Surg 12:413-417.

Bauer CA, Turner JG, Caspary DM, Myers KS, Brozoski TJ (2008) Tinnitus and inferior colliculus activity in chinchillas related to three distinct patterns of cochlear trauma. J Neurosci Res $86: 2564-2578$.

Bilak M, Kim J, Potashner SJ, Bohne BA, Morest DK (1997) New growth of axons in the cochlear nucleus of adult chinchillas after acoustic trauma. Exp Neurol 147:256-268.

Browne CJ, Morley JW, Parsons CH (2012) Tracking the expression of excitatory and inhibitory neurotransmission-related proteins and neuroplasticity markers after noise induced hearing loss. PLoS One 7:e33272.

Cave KM, Cornish EM, Chandler DW (2007) Blast injury of the ear: clinical update from the global war on terror. MilMed 172:726-730.

Chambers AR, Resnik J, Yuan Y, Whitton JP, Edge AS, Liberman MC, Polley DB (2016) Central Gain Restores Auditory Processing following Near-Complete Cochlear Denervation. Neuron 89:867879.

Chen GD, Jastreboff PJ (1995) Salicylate-induced abnormal activity in the inferior colliculus of rats. HearRes 82:158-178. 
Eggermont JJ (2000) Sound-induced synchronization of neural activity between and within three auditory cortical areas. J Neurophysiol 83:2708-2722.

Eggermont JJ (2007) Correlated neural activity as the driving force for functional changes in auditory cortex. HearRes 229:69-80.

Eggermont JJ, Roberts LE (2004) The neuroscience of tinnitus. Trends Neurosci 27:676-682.

Eggermont JJ, Tass PA (2015) Maladaptive neural synchrony in tinnitus: origin and restoration. Frontiers in neurology 6:29.

Finlayson PG, Kaltenbach JA (2009) Alterations in the spontaneous discharge patterns of single units in the dorsal cochlear nucleus following intense sound exposure. HearRes 256:104-117.

Geckle L, Lee R (2004) Soldier perceptions of deployment environmental exposures. In: Force Health Protection Conference Albuquerque, NM.

Henry JA, Dennis KC, Schechter MA (2005) General review of tinnitus: prevalence, mechanisms, effects, and management. JSpeech Lang HearRes 48:1204-1235.

Hughes LF, Turner JG, Parrish JL, Caspary DM (2010) Processing of broadband stimuli across A1 layers in young and aged rats. Hear Res 264:79-85.

Jastreboff PJ (1990) Phantom auditory perception (tinnitus): mechanisms of generation and perception. NeurosciRes 8:221-254.

Jury MA, Flynn MC (2001) Auditory and vestibular sequelae to traumatic brain injury: a pilot study. NZMedJ 114:286-288.

Kaltenbach JA (2011) Tinnitus: Models and mechanisms. Hear Res 276:52-60.

Kimura M, Eggermont JJ (1999) Effects of acute pure tone induced hearing loss on response properties in three auditory cortical fields in cat. HearRes 135:146-162.

Krishnan A, Plack CJ (2009) Auditory brainstem correlates of basilar membrane nonlinearity in humans. AudiolNeurootol 14:88-97. 
Legendy CR, Salcman M (1985) Bursts and recurrences of bursts in the spike trains of spontaneously active striate cortex neurons. JNeurophysiol 53:926-939.

Liberman MC, Kiang NY (1978) Acoustic trauma in cats. Cochlear pathology and auditory-nerve activity. Acta oto-laryngologica Supplementum 358:1-63.

Llano DA, Turner J, Caspary DM (2012) Diminished cortical inhibition in an aging mouse model of chronic tinnitus. J Neurosci 32:16141-16148.

Luo H, Pace E, Zhang X, Zhang J (2014a) Blast-induced tinnitus and spontaneous activity changes in the rat inferior colliculus. Neurosci Lett 580C:47-51.

Luo H, Pace E, Zhang X, Zhang J (2014b) Blast-Induced tinnitus and spontaneous firing changes in the rat dorsal cochlear nucleus. J Neurosci Res 92:1466-1477.

Mao JC, Pace E, Pierozynski P, Kou Z, Shen Y, Vandevord P, Haacke EM, Zhang X, Zhang J (2012) Blast-induced tinnitus and hearing loss in rats: behavioral and imaging assays. Journal of neurotrauma 29:430-444.

Mulders WH, Ding D, Salvi R, Robertson D (2011) Relationship between auditory thresholds, central spontaneous activity, and hair cell loss after acoustic trauma. J Comp Neurol 519:2637-2647.

Mulders WH, Robertson D (2013) Development of hyperactivity after acoustic trauma in the guinea pig inferior colliculus. Hear Res 298:104-108.

Norena AJ, Eggermont JJ (2003) Changes in spontaneous neural activity immediately after an acoustic trauma: implications for neural correlates of tinnitus. HearRes 183:137-153.

Norena AJ, Eggermont JJ (2005) Enriched acoustic environment after noise trauma reduces hearing loss and prevents cortical map reorganization. JNeurosci 25:699-705.

Norena AJ, Eggermont JJ (2006) Enriched acoustic environment after noise trauma abolishes neural signs of tinnitus. Neuroreport 17:559-563.

Norena AJ, Tomita M, Eggermont JJ (2003) Neural changes in cat auditory cortex after a transient puretone trauma. J Neurophysiol 90:2387-2401. 
Luo et al. Blast-induced tinnitus and neural activity changes

1 Richardson BD, Brozoski TJ, Ling LL, Caspary DM (2012) Targeting inhibitory neurotransmission in tinnitus. Brain Res.

3 Roberts LE, Eggermont JJ, Caspary DM, Shore SE, Melcher JR, Kaltenbach JA (2010) Ringing ears: the $4 \quad$ neuroscience of tinnitus. Hear Res 30:14972-14979.

5 Schaette R, Kempter R (2012) Computational models of neurophysiological correlates of tinnitus. Front 6 auditory cortex after localized tone-induced hearing loss. HearRes 180:28-38.

9 Shore SE, Roberts LE, Langguth B (2016) Maladaptive plasticity in tinnitus--triggers, mechanisms and treatment. Nature reviews Neurology 12:150-160.

11 Swadlow HA, Gusev AG (2001) The impact of 'bursting' thalamic impulses at a neocortical synapse. Nat Neurosci 4:402-408.

13 Vogler DP, Robertson D, Mulders WH (2011) Hyperactivity in the ventral cochlear nucleus after cochlear trauma. J Neurosci 31:6639-6645.

15 Wu C, Martel DT, Shore SE (2016) Increased Synchrony and Bursting of Dorsal Cochlear Nucleus Fusiform Cells Correlate with Tinnitus. J Neurosci 36:2068-2073.

17 Zhang J, Luo H, Pace E, Li L, Liu B (2015) Psychophysical and neural correlates of noised-induced tinnitus in animals: Intra- and inter-auditory and non-auditory brain structure studies. Hear Res.

Zhang JS, Kaltenbach JA (1998) Increases in spontaneous activity in the dorsal cochlear nucleus of the rat following exposure to high-intensity sound. Neurosci Lett 250:197-200.

\section{Figure Legends}

23 Figure 1. SFRs recorded in three frequency regions $(2-4 \mathrm{kHz}, 4-16 \mathrm{kHz}$ and $16-42 \mathrm{kHz})$ in the $\mathrm{AC}$ of tinnitus positive, tinnitus negative and age-matched control rats at one day, one 
month, and three months after blast exposure. For the tinnitus positive group, note the significant decrease in 16-42 $\mathrm{kHz}$ SFR at one month after exposure, and the significant increase across all frequency regions at three months after exposure. Error bars represent standard error of the mean. ${ }^{*} p<0.05$.

Figure 2. Plot showing the temporal profile of the effects of blast-induced tinnitus and hearing impairment on SFRs for the control, tinnitus negative and tinnitus positive groups. Error bars represent standard error of the mean. ${ }^{*} p<0.05$.

Figure 3. Bursting activity measured in three frequency regions $(2-4 \mathrm{kHz}, 4-16 \mathrm{kHz}$ and $16-42$ $\mathrm{kHz}$ ) in the $\mathrm{AC}$ of tinnitus positive, tinnitus negative, and age-matched control rats at one day, one month, and three months after blast exposure. Note the significant elevation in bursting rates in the $2-4 \mathrm{kHz}$ region at one month after blast and across all frequency regions at three months after blast. Error bars represent standard error of the mean. ${ }^{*} p<0.05$.

Figure 4. Plot showing the temporal profile of the effects of blast-induced tinnitus and hearing impairment on bursting activity for the control, tinnitus negative and tinnitus positive groups. No statistically significant differences in bursting activity change were found between frequency regions $(\mathrm{p}>0.05)$. Error bars represent standard error of the mean. 
Luo et al.

Blast-induced tinnitus and neural activity changes

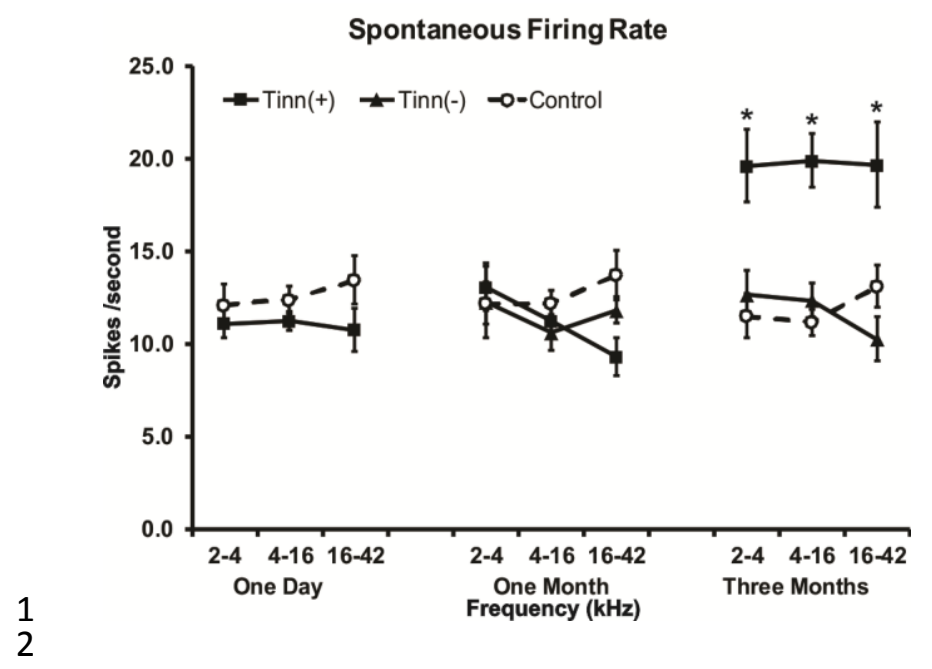


Luo et al.

Blast-induced tinnitus and neural activity changes

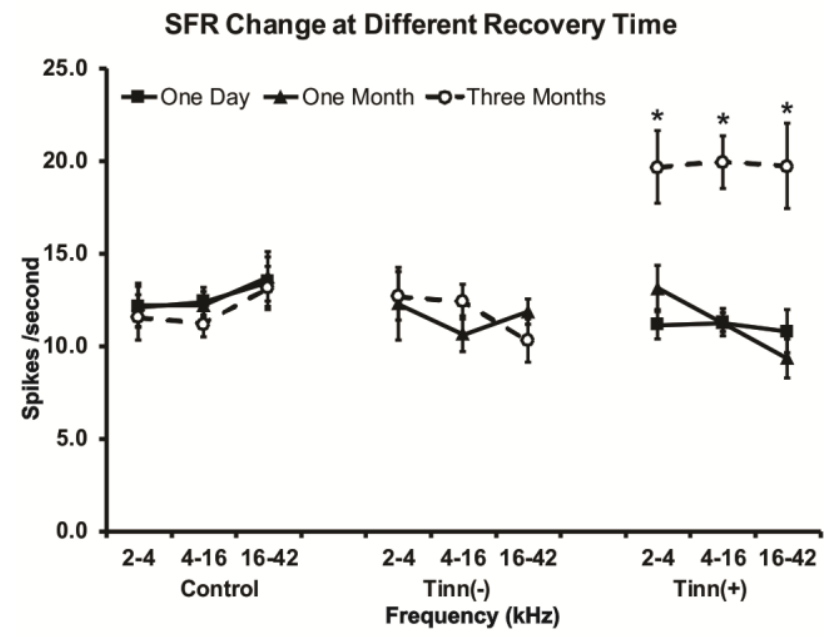

1 


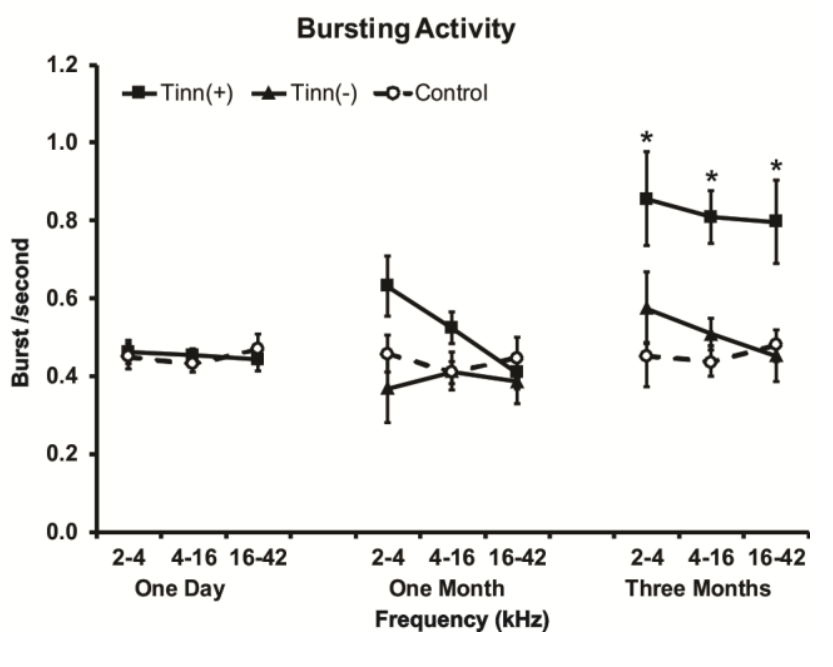


Bursting Activity Change at Different Recovery Time

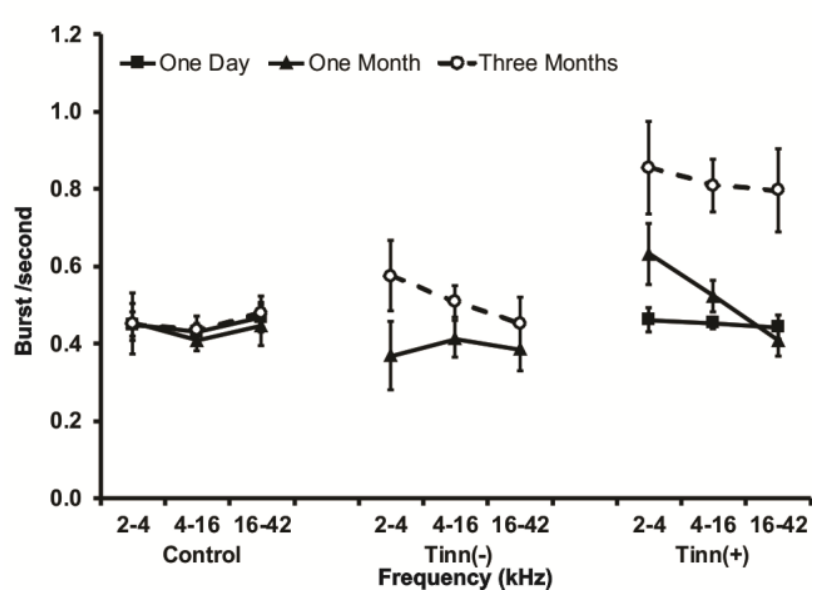

